\title{
MIXED FOLIATE CR-SUBMANIFOLDS IN A COMPLEX HYPERBOLIC SPACE ARE NON-PROPER
}

\author{
BANG-YEN CHEN \\ Department of Mathematics \\ Michigan State University \\ East Lansing, Michigan 48824, USA \\ and \\ BAO-QIANG WU \\ Department of Mathematics \\ Xuzhou Teacher's College \\ Jiangsu, People's Republic of China
}

(Received June 4, 1987)

\begin{abstract}
It was conjectured in [1 II] (also in [2]) that mixed foliate CR-submanifolds in a complex hyperbolic space are either complex submanifolds or totally real aubmanifolds. In this paper we give an affirmative solution to this conjecture.
\end{abstract}

KEY WORDS AND PHRASRS: CR-submanifolds, complex hyperbolic space, mixed foliate. 1980 AMS SUBJECT CLASSIFICATION CODE: 53B25, 53C40.

\section{INTRODUCTION.}

A submanifold $M$ of a Kaehler manifold $\tilde{M}$ is called a CR-submanifold if (1) the maximal complex subspace $D_{X}$ of the tangent space $T_{X} \tilde{M}$ containing in $T_{X} M, x \in M$, defines a differentiable distribution $D$, called the holomorphic distribution, and (2) the orthogonal complementary distribution $\sigma^{+}$of $D$ in $T M$ is a totally real distribution, i.e., $J \sigma_{x}^{+} \subset T_{x}^{M}$, where $J$ denotes the almost complex structure of $\tilde{M}$ and $T_{x}^{M}$ the normal space of $M$ at $x$. Complex submanifolds and totally real submanifolds of $\tilde{M}$ are trivial examples of CR-submanifolds. A CR-submanifold is called proper if it is neither a complex submanifold nor a totally real submanifold. The totally real distribution $\delta^{+}$of a CR-submanifold of a Kaehler manifold is always integrable [1,3]. A CR-submanifold $M$ is called mixed foliate if (a) the holomorphic distribution $D$ is integrable, and (b) the second fundamental form $\sigma^{0}$ of $M \quad$ in $\tilde{M}$ satisfies $\sigma^{0}\left(0,0^{+}\right)=\{0\}$.

It is known that mixed foliate CR-submanifolds in $c^{m}$ are exactly CR-products in cm [1 I] and mixed foliate CR-submanifolds in CPm are non-proper [4]. It was conjectured in [1 11$]$ (also in [2]) that mixed foliate CR-submanifolds in a comnlev hyperbolic space $H^{m}$ are non-proper too. 
In this paper, we solve this conjecture completely to give the following

THEOREM 1. Let $M$ be a mixed foliate CR-submanifold of $\mathrm{H}^{\mathrm{m}}$. Then $\mathrm{M}$ is either a complex submanifold or a totally real submanifold.

\section{PRELIMINARIES.}

For simplicity, we assume that $\mathrm{H}^{\mathrm{m}}$ is the (complex) m-dimensional complex hyperbolic space with constant holomorphic sectional curvature -4. Let $M$ be a mixed foliate CR-submanifold of $\mathrm{H}^{\mathrm{m}}$. Then, by definition, the holomorphic distribution $D$ of $M$ is integrable and the second fundamental form $\sigma^{\circ}$ of $M$ in $\mathrm{H}^{\mathrm{m}}$ eatisfies $\sigma^{\circ}\left(0,0^{+}\right)=\{0\}$. We denote by $\langle$,$\rangle the metric tensor of \mathrm{H}^{\mathrm{m}}$ as well as the induced one on $M$. Let $D^{0}$ and $A^{0}$ denote the normal connection and the Weingarten map of $M$ in $\mathrm{H}^{\mathrm{m}}$, respectively. If $\mathrm{N}$ is a leaf of $D$, then $N$ is a complex submanifold of $\mathrm{H}^{\mathrm{m}}$. Denote by $\sigma, D, A$ and $\nabla$ the second fundamental form, the normal connection, the Weingarten map and the Levi-Civita connection of $N\left(i n H^{m}\right)$, respectively, and by $\sigma^{\prime}, D^{\prime}, A^{\prime}$ the corresponding quantities for $N$ in $M$. Then we have $\sigma(X, Y)=\sigma^{\prime}(X, Y)+\sigma^{\circ}(X, Y)$ for $X, Y$ tangent to $N$. Since $\sigma^{\circ}\left(0_{,} \sigma^{+}\right)=\{0\}$, we also have $A J Z=A 9 Z$, on $T N$, for $Z$ in $\sigma^{t}$. Since $N$ is a complex submanifold of $\mathrm{H}^{\mathrm{m}}$, the almost complex structure $\mathrm{J}$ satisfies $\sigma(\mathrm{JX}, \mathrm{Y})=\mathrm{J} \sigma(\mathrm{X}, \mathrm{Y})=\sigma(\mathrm{X}, \mathrm{JY}), \mathrm{AJ}_{\mathrm{J}}=$ $J_{\xi}, J A \xi=-A_{\xi} J$, for $X, Y$ tangent to $N$ and $\xi$ normal to $N$.

For any vector $X$ tangent to $M$, we put JX $=$ PX + FX where PX and FX are the tangential and the normal components of $J X$, respectively. For a vector $\xi$ normal to $M$, we put $J \xi=t \xi+f \xi$, where $t \xi$ and $f \xi$ are the tangential and the normal components of $J \xi$, respectively.

Since $H^{\mathrm{m}}$ is of constant holomorphic sectional curvature -4 , the curvature tensor $\tilde{\mathbf{R}}$ of $\mathbf{H}^{\mathrm{m}}$ is given by

$$
\begin{aligned}
\tilde{\mathbf{R}}(\mathbf{X}, \mathbf{Y}) \mathbf{Z}=\langle\mathbf{X}, \mathbf{Z}\rangle \mathbf{Y}-\langle\mathbf{Y}, \mathbf{Z}\rangle \mathbf{X}+\langle\mathbf{J X}, \mathbf{Z}\rangle \mathbf{J Y} \\
-\langle\mathbf{J Y}, \mathbf{Z}\rangle \mathbf{J X}-\mathbf{2}\langle\mathbf{X}, \mathbf{J Y}\rangle \mathbf{J Z}
\end{aligned}
$$

for $X, Y, Z$ tangent to $\mathrm{H}^{\mathrm{m}}$.

We need the following result of [1 II] for later use.

IneM 1. Let $M$ be $a$ mixed foliate CR-submanifold of IP. Then

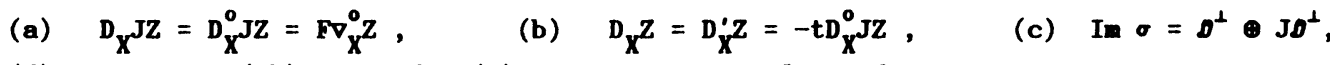
(d) $A_{Z}, \Lambda_{J Z} \in O(2 h)$, and (e) $A_{Z} A_{W}+A_{W} A_{Z}=0$, for $X$ tangent to $N$ and orthonoreal vectors $\mathrm{Z}$ and $\mathrm{N}$ in $\mathrm{de}$.

LEMMA 2. Under the hypothesis of Lemma 1, if $M$ is proper, then (a) each leaf $\mathrm{N}$ of $\mathrm{D}$ lies in a complex $(h+p)$-dimensional totally geodesic complex submanifold $\mathrm{H}^{\mathrm{h}+\mathrm{p}}$ of $\mathrm{H}^{\mathrm{m}}$ and $(\mathrm{b}) \mathrm{h}+1 \otimes \mathrm{p} \otimes 2$ and $\mathrm{h} \gg 2$ where $\mathrm{p}=\operatorname{dim}_{\mathrm{R}} \boldsymbol{\sigma}^{+}$ and $h=\operatorname{dim}_{\mathbf{c}} \boldsymbol{D}$.

\section{MORE LEMMAS.}

Let $M$ be a mixed foliate CR-submanifold of $H^{m}$. If $M$ is non-proper, there is nothing to prove. Thus we may assume that $M$ is proper. By Lemma $2, p \geq 2$. From Lemma 1, we have

$$
A_{z} A_{w}+A_{w} A_{z}=0
$$

for orthonormal vectors $\mathrm{Z}, \mathrm{W}$ in $\boldsymbol{\sigma}^{+}$. Let $Z_{1}, \ldots, Z_{p}$ be an orthonormal frame of $\delta^{\perp}$. We put 


$$
\Lambda_{\alpha}=\Lambda_{Z_{\alpha}}, \quad \Lambda_{\alpha *}=A_{J Z_{\alpha}}, \quad \alpha=1, \ldots, p
$$

From property (d) of Lemma 1, each $A_{a^{*}}$ has eigenvalues 1 and -1 with the game multiplicity $h$. Let $X_{1}, \ldots, X_{h}$ be $h$ orthonormal eigenvectors of $A_{\alpha *}$ with eigenvalue 1. Then $\mathrm{JX}_{1}, \ldots, \mathrm{JX} \mathrm{X}_{\mathrm{p}}$ are eigenvectors of $\Lambda_{\alpha *}$ with eigenvalue -1 . With

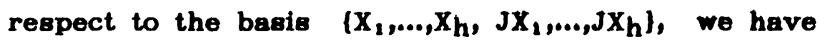

$$
A_{\alpha *}=\left(\begin{array}{cc}
I_{h} & 0 \\
0 & -I_{h}
\end{array}\right), \quad J=\left(\begin{array}{cc}
0 & -I_{h} \\
& \\
I_{h} & 0
\end{array}\right) \text {, }
$$

where Ih denotes the $h \times h$ identity matrix. Thus, by (2.1), we have

$$
A_{\alpha}=\left(\begin{array}{rr}
0 & -I_{h} \\
-I_{h} & 0
\end{array}\right)
$$

In particular, if we choose $\alpha=1$, we obtain

$$
\Lambda_{1}=\left(\begin{array}{cc}
0 & -I_{h} \\
-I_{h} & 0
\end{array}\right), \quad A_{1 *}=\left(\begin{array}{cc}
I_{h} & 0 \\
0 & -I_{h}
\end{array}\right) \text {. }
$$

From (2.1) and (3.1) we have

$$
\Lambda_{\alpha} \Lambda_{\beta *}-\Lambda_{\beta * A_{\alpha}}=0, \quad \alpha \neq \beta, \quad \alpha, \beta=1, \ldots, p \text {. }
$$

Using (3.1), (3.5) and (3.6) we way get

$$
A_{2}=\left(\begin{array}{cc}
B & 0 \\
0 & -B
\end{array}\right), \quad A_{2 *}=\left(\begin{array}{cc}
0 & B \\
B & 0
\end{array}\right) \text {. }
$$

Since $A_{2} \in O(2 h)$ (Lema 1), we also have

$$
B \in O(h), \quad t_{B}=B,
$$

where $t_{B}$ denotes the transpose of $B$.

LEMIA 3. If $M$ is a proper mixed foliate $C R$-submanifold of $\mathrm{H}^{\mathrm{m}}$, then $\mathrm{p} 3$.

PROOF. Under the hypothesie, Lemma 2 showe that if $p<3$, then $p=2$. If $p=2$, we may choose an orthonormal frame $X_{1}, \ldots, X_{h}, J X_{1}, \ldots, J X_{h}, Z_{1}, Z_{2}, J Z_{1}, J Z_{2}$ such that, with respect to this frame, $A_{1}, A_{2}, A_{1} *$ and $A_{2} *$ take the forms of (3.5), (3.7) and (3.8).

We put

$$
V=\operatorname{span}\left\{X_{1}, \ldots, X_{h}\right\}
$$

Then $T N=V \oplus J V$. Since $B \in O(h)$ with $t_{B}=B$, we may further choose $\left\{X_{1}, \ldots, X_{h}\right\}$ such that with respect to it, $B$ has the form:

$$
B=\left(\begin{array}{cc}
I_{\mathbf{r}} & 0 \\
0 & -\mathbf{I}_{\mathbf{h}-\mathbf{r}}
\end{array}\right)
$$


CASB 1: $r=h$. In this case we have

$$
-\Lambda_{1}=\Lambda_{2 *}=\left(\begin{array}{cc}
0 & I_{h} \\
I_{h} & 0
\end{array}\right), \quad \Lambda_{1 *}=\Lambda_{2}=\left(\begin{array}{cc}
I_{h} & 0 \\
0 & -I_{h}
\end{array}\right) .
$$

So, if we put

$$
w=\frac{1}{\sqrt{2}}\left(\mathrm{Z}_{1}+\mathrm{JZ}_{2}\right)
$$

then $A_{W}=\Lambda_{J W}=0$, which contradicts statement (c) of Lensa $l$.

CASE 2: $r=0$. This case is impossible by applying an argument similar to Case 1.

CASE 3: $r>0$ and $h>r$. In this case we can decompose $v$ and JV into orthogonal decompositions:

$$
\mathbf{V}=\mathbf{V}^{\prime} \oplus \mathrm{V}^{\prime \prime}, \quad \mathrm{JV}=\mathrm{JV}^{\prime} \oplus \mathrm{J} \mathrm{V}^{\prime \prime},
$$

where $V^{\prime}$ and $V^{\prime \prime}$ are eigenspaces of $B$ (defined by (3.10)) with eigenvalues 1 and -1 , respectively. By (3.5), (3.7), (3.10) and Lemma 1 we have

$$
\begin{aligned}
& \sigma(\mathrm{X}, \mathrm{T})=\langle\mathrm{JX}, \mathrm{T}\rangle\left(\mathrm{JZ}_{2}-\mathrm{Z}_{1}\right)+\langle\mathrm{X}, \mathrm{T}\rangle\left(\mathrm{JZ}_{1}+\mathrm{Z}_{2}\right), \\
& \sigma(\mathrm{Y}, \mathrm{T})=-\langle\mathrm{JY}, \mathrm{T}\rangle\left(\mathrm{JZ}_{2}+\mathrm{Z}_{1}\right)+\langle\mathrm{Y}, \mathrm{T}\rangle\left(\mathrm{JZ}_{1}-\mathrm{Z}_{2}\right)
\end{aligned}
$$

for $X \in V^{\prime}, \quad Y \in V^{\prime \prime}$ and $T \in T N$.

By Lemma 1 we have

$$
\mathrm{DZ}_{1}=\lambda \mathrm{Z}_{2}, \quad \mathrm{DZ}_{2}=-\lambda \mathrm{Z}_{1}, \quad \mathrm{DJZ}_{1}=\lambda \mathrm{JZ}_{2}, \quad \mathrm{DJZ}_{2}=-\lambda \mathrm{JZ}_{1} \text {, }
$$

for some 1-form $\lambda$ on $N$. Since $N$ is a complex submanifold of $H^{m}$, the equation of Codazzi gives

$$
\left(\bar{\nabla}_{\mathbf{X}} \boldsymbol{\sigma}\right)(\mathbf{Y}, \mathbf{Z})=\left(\bar{\nabla}_{\mathbf{Y}} \boldsymbol{\sigma}\right)(\mathbf{X}, \mathbf{Z})
$$

where $\left(\bar{\nabla}_{X} \sigma\right)(Y, Z)=D_{X} \sigma(Y, Z)-\sigma\left(\nabla_{X} Y, Z\right)-\sigma\left(Y, \nabla_{X} Z\right)$ for $X, Y, Z$ tangent to $N$.

In particular, if $X \in V^{\prime}, Y \in V^{*}$ and $W \in J V^{\prime}$, then by applying (3.14), (3.15) and (3.16), we see that the $Z_{2}$-components of both sides of (3.16) yield

$$
0=\lambda(Y)\langle J X, W\rangle-\left\langle W, \nabla_{Y} X\right\rangle+\left\langle X, \nabla_{Y} W\right\rangle \text {. }
$$

Because $\langle X, W\rangle=0, \quad(3.17)$ implies

$$
2\langle\nabla Y X, W\rangle=\lambda(Y)\langle J X, W\rangle \text {. }
$$

Similarly, if $X \in V^{\prime}, Y \in V^{\prime \prime}$ and $W^{\prime} \in J^{\prime}$, the $J_{1}$-components yield

$$
2\langle\nabla Y X, W\rangle-\lambda(Y)\langle J X, W\rangle=2\langle\nabla X Y, W\rangle \text {. }
$$

Combining (3.18) and (3.19) we find

$$
\langle\nabla \mathbf{X} \mathbf{Y}, \boldsymbol{W}\rangle=\mathbf{0}
$$

which also implies $\langle\nabla \mathrm{XW}, \mathrm{Y}\rangle=0$. Therefore

$$
\nabla V^{\prime} V^{\prime \prime} \perp J V^{\prime}, \quad \nabla V^{\prime} J^{\prime} \perp V^{\prime \prime} .
$$

Since $J$ is parallel, this also gives

$$
\nabla V^{\prime} J V^{\prime \prime} \perp V^{\prime}, \quad \nabla V^{\prime} V^{\prime} \perp J V^{\prime \prime} .
$$


Similarly, we may obtain

$$
\begin{array}{ll}
\nabla V^{\prime} V^{\prime}+V^{\prime \prime}, & \nabla V^{\prime} J V^{\prime}+J V^{\prime \prime}, \\
\nabla V^{\prime} V^{\prime \prime}+V^{\prime}, & \nabla V^{\prime} J V^{\prime \prime}+J V^{\prime} .
\end{array}
$$

Let $U^{\prime}=V^{\prime} \oplus J^{\prime}$ and $U^{\prime \prime}=V^{\prime} \oplus J V^{\prime \prime}$. Then $(3.21)-(3.24)$ show that

$$
\nabla \mathbf{V} \cdot \mathbf{U}^{\prime}+\mathbf{U}^{\prime \prime}, \quad \nabla \mathbf{V} \cdot \mathbf{U}^{\prime \prime}+\mathbf{U}^{\prime}
$$

In a similar way we may also obtain $\nabla \mathrm{JV} \mathrm{U}^{\prime} \perp \mathrm{U}^{\prime \prime}$ and $\nabla \mathrm{JV} \mathrm{U}^{\prime \prime} \perp \mathrm{U}^{\prime}$. Therefore, we see that $U^{\prime}$ and $U^{\prime \prime}$ are both integrable and parallel distributions. Thus $N$ is locally the Riemannian product of two Kaehler manifolds. This is a contradiction since $H^{\mathrm{m}}$ admits no complex submanifold which is a product of two Kaehler manifolds (cf. [l 1 I $]$ ).

LEMMA 4. Let $M$ be a proper mixed foliate CR-submanifold of $\mathrm{H}^{\mathrm{m}}$ If $p=\operatorname{dim}_{R} \theta^{\prime}, 3$, then $h=\operatorname{dim} \theta=2 r$ is oven and with respect to a suitable

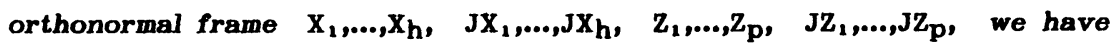

$$
\begin{aligned}
& \mathbf{A}_{1}=\left(\begin{array}{cc}
0 & -I_{h} \\
-I_{h} & 0
\end{array}\right), \quad A_{1 *}=\left(\begin{array}{cc}
I_{h} & 0 \\
0 & -I_{h}
\end{array}\right) \text {, } \\
& \mathbf{A}_{2}=\left(\begin{array}{cc}
B & 0 \\
0 & -B
\end{array}\right), \quad A_{2 *}=\left(\begin{array}{cc}
0 & B \\
B & 0
\end{array}\right), \quad B=\left(\begin{array}{cc}
I_{r} & 0 \\
0 & -I_{r}
\end{array}\right) \text {, } \\
& A_{3}=\left(\begin{array}{cc}
C & 0 \\
0 & -C
\end{array}\right), \quad A_{3 *}=\left(\begin{array}{cc}
0 & C \\
C & 0
\end{array}\right), \quad C=\left(\begin{array}{ll}
0 & I_{r} \\
I_{r} & 0
\end{array}\right) \text {. }
\end{aligned}
$$

If $p>4$, then, for $\alpha \geq 4$, we also have

$$
A_{\alpha}=\left(\begin{array}{cc}
D_{\alpha} & 0 \\
0 & -D_{\alpha}
\end{array}\right), \quad A_{\alpha *}=\left(\begin{array}{cc}
0 & D_{\alpha} \\
& \\
D_{\alpha} & 0
\end{array}\right), \quad D_{\alpha}=\left(\begin{array}{cc}
0 & B_{\alpha} \\
t_{B_{\alpha}} & 0
\end{array}\right)
$$

for some $\mathrm{E}_{\alpha} \in O(\mathrm{r})$ such that $t_{\mathrm{E}_{\alpha}}=-\mathrm{E}_{\alpha}$.

ProOr. Under the hypothesis, there is a suitable orthonormal frame $X_{1}, \ldots, X_{h}$, $\mathrm{JX}_{1}, \ldots, \mathrm{JX}_{\mathrm{h}}, \mathrm{Z}_{1}, \ldots, \mathrm{Z}_{\mathrm{p}}, \mathrm{JX}_{1}, \ldots, \mathrm{JX} \mathrm{p}$ such that $\mathrm{A}_{1}, \mathrm{~A}_{2}, \mathrm{~A}_{1} *$ and $\mathrm{A}_{2} *$ take the desired forms (cf. (3.5), (3.7) and (3.10)). Since $A_{\alpha} A_{1}+A_{1} A_{\alpha}=0$ for $\alpha \backslash 3$, we also have

$$
A_{\alpha}=\left(\begin{array}{cc}
D_{\alpha} & 0 \\
0 & -D_{\alpha}
\end{array}\right), \quad A_{\alpha *}=\left(\begin{array}{cc}
0 & D_{\alpha} \\
& \\
D_{\alpha} & 0
\end{array}\right) \text {, }
$$

where $D_{\alpha} \in O(h)$ with $t_{D_{\alpha}}=D_{\alpha}$. From Lemma 1 we also have

$$
A_{2} A_{\alpha}+A_{\alpha} A_{2}=0, \quad A_{2} A_{\alpha *}-A_{\alpha * A_{2}}=0 .
$$

From this we see that each $D_{\alpha}$ takes the following form:

$$
D_{\alpha}=\left(\begin{array}{cc}
0 & B_{\alpha} \\
t_{B_{\alpha}} & 0
\end{array}\right), \quad \alpha \geqslant 3,
$$

where each $E_{\alpha}$ is a $(r \times(h-r))$-matrix. Since $D_{\alpha} \in O(h)$, this implies 


$$
B_{\alpha} t_{E_{\alpha}}=I_{r} \quad \text { and } \quad t_{B_{\alpha} B_{\alpha}}=I_{h-r} .
$$

It is clear that this is impossible unless $E_{\alpha}$ is a square matrix. Therefore, we have $r=0, h=r$, or $h=2 r$. However, the first two cases cannot occur since, for instance, if $r=0$, then $A_{2}=-A_{1} *$ which implies $A_{\alpha}=0$ by virture of (3.30). This contradicts to (c) of Lemma 1. Similar argument works for the second case. Consequently, $h=2 r$ which is even. Now, let $X_{1}, \ldots, X_{h}$ be chosen in such a way that

$$
\mathbf{X}_{\mathrm{r}+1}=\mathrm{A}_{3} \mathrm{X}_{1}, \ldots, \mathrm{X}_{\mathrm{h}}=\mathrm{A}_{3} \mathbf{X}_{\mathbf{r}} \text {. }
$$

Then $A_{3}$ and $A_{3} *$ are expressed in the forms given in (3.31). Finally, for each $\alpha \geq 4$, by using the properties $A_{3} A_{\alpha}+A_{\alpha} A_{3}=0$ and $D_{\alpha} \in O(h)$, we may conclude that $D_{\alpha}$ is in the desired form.

(Q.E.D.)

LEMMA 5. Let $M$ be a proper mixed foliate CR-submanifold of $\mathrm{H}^{\mathrm{m}}$. If $\mathrm{p}$, then $h \geq 2 p-4$. Furthermore, we may choose the orthonormal frame such that, in addition to (3.27) and (3.28), we also have

$$
\begin{gathered}
\Lambda_{\alpha} \Lambda_{3} X_{1}=X_{\alpha-2}, \quad \Lambda_{\alpha} X_{1}=-X_{r+\alpha-2}, \quad p \geqslant \alpha \geqslant 4, \\
Y_{i}=X_{r+i}=A_{3} X_{i}, \quad i=1, \ldots, r .
\end{gathered}
$$

PROOF. As given in the proof of Lemma 3, we decompose the tangent bundle of $N$ into orthogonal decomposition:

$$
\mathrm{TN}=\mathrm{V} \oplus \mathrm{JV}, \quad \mathrm{V}=\mathrm{V}^{\prime} \oplus \mathrm{V}^{\prime \prime}, \quad \mathrm{JV}=\mathrm{JV}^{\prime} \oplus \mathrm{JV}^{*}
$$

Such a decomposition is given with respect to $A_{1} *$ and $A_{2}$. Now, let $X_{1}$ be a unit vector in $V^{\prime}$. We put $Y_{1}=X_{r+1}=A_{3} X_{1}$ as before. Then (e) of Lemma 1 implies that $A_{3} Y_{1}, \ldots, A_{p} Y_{1}$ are orthonormal vectors in $V^{\prime}$ (cf. p. 500 of [4 II]). From this we conclude that $r \backslash p-2$ which is equivalent to $h \backslash 2 p-4$. Now, we put

$$
\begin{gathered}
X_{i}=A_{i+2} A_{3} X_{1}=A_{i+2} Y_{1}, 2 \leftarrow i \leqslant 2, \\
Y_{i}=X_{r+i}=A_{3} X_{i}, \quad \text { for } i=2, \ldots, p-2, \ldots, r .
\end{gathered}
$$

Then, (3.27) holds. Since

$$
\Lambda_{\alpha} X_{1}=\Lambda_{\alpha} \Lambda_{3} Y_{1}=-\Lambda_{3} \Lambda_{\alpha} Y_{1}=-\Lambda_{3} X_{\alpha-2}=-Y_{\alpha-2},
$$

we also have (3.33). Formulas (3.34) are nothing but (3.37).

(Q.E.D.)

From properties (a) and (b) of Lemma 1, we have

$$
\mathrm{DZ}_{\alpha}=\sum_{\beta=1}^{\mathrm{p}} \boldsymbol{\theta}_{\alpha \beta} \mathrm{Z}_{\beta}, \quad \boldsymbol{\theta}_{\alpha \beta}=\boldsymbol{\theta}_{\beta \alpha}, \quad \alpha, \beta=1, \ldots, \mathrm{p} .
$$

for some 1-forms $\theta_{\alpha \beta}$ on $N$. (3.39) gives

$$
\mathrm{DJZ}_{\alpha}=\sum_{\beta} \boldsymbol{\theta}_{\alpha \beta} \mathrm{JZ}_{\beta}
$$

Invin 6. Under the hypothesis and the notations of Lema 5, we have

$$
\begin{aligned}
& 2\left\langle\nabla_{\mathrm{T}} \mathrm{X}_{j}, J X_{k}\right\rangle=\delta_{j k} \theta_{12}(T), \\
& 2\left\langle\nabla_{\mathrm{T}} \mathrm{Y}_{j}, J Y_{k}\right\rangle=\delta_{j k} \theta_{21}(T), \\
& 2\left\langle\nabla_{\mathrm{T}} X_{j}, J Y_{k}\right\rangle=\delta_{j k} \theta_{13}(T)+\sum_{\alpha \geq 4}\left\langle A_{\alpha} X_{j}, Y_{k}\right\rangle \theta_{1 \alpha}(T),
\end{aligned}
$$




$$
\begin{aligned}
& 2\left\langle\nabla_{\mathrm{T}} \mathrm{X}_{\mathrm{j}}, \mathrm{Y}_{\mathrm{k}}\right\rangle=\delta_{\mathrm{jk} \boldsymbol{\theta}_{23}}(\mathrm{~T})+\sum_{\alpha \neq 4}\left\langle\mathrm{~A}_{\alpha} \mathrm{X}_{\mathrm{j}}, \mathrm{Y}_{\mathbf{k}}\right\rangle \boldsymbol{\theta}_{2 \alpha}(\mathrm{T}), \\
& \left\langle\nabla_{\mathrm{T}} \mathrm{Y}_{\mathrm{i}}, \mathrm{Y}_{\mathbf{k}}\right\rangle-\left\langle\nabla_{\mathrm{T}} \mathrm{X}_{\mathrm{i}}, \mathrm{X}_{\mathrm{k}}\right\rangle=\sum_{\alpha \neq 4}\left\langle\Lambda_{\alpha} \mathrm{X}_{\mathrm{i}}, \mathrm{Y}_{\mathbf{k}}\right\rangle \boldsymbol{\theta}_{3 \alpha}(\mathrm{T})
\end{aligned}
$$

for $\mathrm{T}$ tangent to $\mathrm{N}$.

PROOF. The proof of this lemma is based mainly on the equation of Codazzi. Let $X_{1}, \ldots, X_{r}, Y_{1}, \ldots, Y_{r}$ be an orthonormal frame of $V^{\prime} \oplus V^{\prime \prime}=V$ with $Y_{i}=X_{r+i}=A_{3} X_{i}$ as before, then for any vector $T$ tangent to $N$, Lemma 4 gives

$$
\begin{aligned}
& \sigma\left(X_{i}, T\right)=\left\langle J X_{i}, T\right\rangle\left(J Z_{1}-Z_{1}\right)+\left\langle X_{i}, T\right\rangle\left(Z_{2}+J Z_{1}\right) \\
& +\left\langle\mathrm{Y}_{\mathrm{i}}, \mathrm{T}\right\rangle \mathrm{Z}_{3}+\left\langle\mathrm{JY}_{\mathrm{i}}, \mathrm{T}\right\rangle \mathrm{JZ}_{3}+\sum_{\alpha>4}\left(\left\langle\mathrm{~A}_{\alpha} \mathrm{X}_{\mathrm{i}}, \mathrm{T}\right\rangle \mathrm{Z}_{\alpha}+\left\langle\mathrm{A}_{\alpha \star} \mathrm{X}_{\mathrm{i}}, \mathrm{T}\right\rangle \mathrm{JZ}_{\alpha}\right) \\
& \sigma\left(Y_{i}, T\right)=-\left\langle J Y_{i}, T\right\rangle\left(J Z_{2}+Z_{1}\right)-\left\langle Y_{i}, T\right\rangle\left(Z_{2}-J Z_{1}\right) \\
& +\left\langle\mathrm{X}_{\mathrm{i}}, \mathrm{T}\right\rangle \mathrm{Z}_{3}+\left\langle\mathrm{JX}_{\mathrm{i}}, \mathrm{T}\right\rangle \mathrm{JZ}_{3}+\sum_{\alpha \geqslant 4}\left(\left\langle\mathrm{~A}_{\alpha} \mathrm{Y}_{\mathrm{i}}, \mathrm{T}\right\rangle \mathrm{Z}_{\alpha}+\left\langle\mathrm{A}_{\alpha *} \mathrm{Y}_{\mathrm{i}}, \mathrm{T}\right\rangle \mathrm{JZ}_{\alpha}\right) .
\end{aligned}
$$

.From (3.46), (3.47), (2.3) and Lemmas 4 and 5, we obtain

$$
\begin{aligned}
& \left(\bar{\nabla}_{\mathbf{X}_{\mathbf{i}}}{ }\right)\left(\mathrm{JY}_{\mathbf{j}}, \mathrm{JY}_{\mathbf{k}}\right)=\mathrm{D}_{\mathbf{X}_{\mathbf{i}}}\left(\delta_{\mathbf{j k}} \mathbf{Z}_{\mathbf{2}}-\boldsymbol{\delta}_{\mathbf{j k}} \mathrm{JZ}_{\mathbf{1}}\right)-\left\langle\mathrm{JY}_{\mathbf{k}}, \nabla_{\mathbf{X}_{\mathbf{i}}} \mathbf{Y}_{\mathbf{j}}\right\rangle\left(\mathrm{JZ}_{\mathbf{2}}+\mathrm{Z}_{\mathbf{1}}\right) \\
& -\left\langle\mathbf{Y}_{\mathbf{k}}, \boldsymbol{\nabla}_{\mathbf{X}_{\mathbf{i}}} \mathbf{Y}_{\mathbf{j}}\right\rangle\left(\mathbf{Z}_{\mathbf{2}}-\mathbf{J Z _ { 1 }}\right)+\left\langle\mathbf{X}_{\mathbf{k}}, \boldsymbol{\nabla} \mathbf{X}_{\mathbf{i}} \mathbf{Y}_{\mathbf{j}}\right\rangle \mathbf{Z}_{\mathbf{3}}+\left\langle J \mathbf{X}_{\mathbf{k}}, \nabla_{\mathbf{X}_{\mathbf{i}}} \mathbf{Y}_{\mathbf{j}}\right\rangle J Z_{3} \\
& +\sum_{\alpha \geqslant 4}\left(\left\langle A_{\alpha} Y_{k}, \nabla_{X_{i}} Y_{j}\right\rangle Z_{\alpha}+\left\langle A_{\alpha} * Y_{k}, \nabla_{X_{i}} Y_{j}\right\rangle J Z_{\alpha}\right) \\
& -\left\langle J Y_{j}, \nabla_{\mathbf{X}_{i}} \mathbf{Y}_{\mathbf{k}}\right\rangle\left(J Z_{\mathbf{2}}+Z_{\mathbf{l}}\right)-\left\langle\mathbf{Y}_{j}, \nabla_{\mathbf{X}_{\mathbf{i}}} \mathbf{Y}_{\mathbf{k}}\right\rangle\left(\mathbf{Z}_{\mathbf{2}}-J Z_{\mathbf{l}}\right)
\end{aligned}
$$

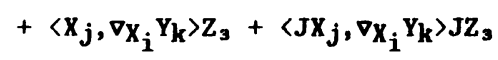

$$
\begin{aligned}
& +\sum_{\alpha \geq 4}\left(\left\langle\Lambda_{\alpha} Y_{j}, \nabla X_{i} Y_{k}\right\rangle Z_{\alpha}+\left\langle\Lambda_{\alpha *} Y_{j}, \nabla X_{i} Y_{k}\right\rangle J Z_{\alpha}\right)
\end{aligned}
$$

Moreover, from (3.46), (3.47) and Lewas 4 and 5, we also obtain

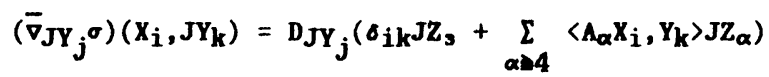

$$
\begin{aligned}
& +\left\langle Y_{k}, \nabla_{J} Y_{j} X_{i}\right\rangle\left(J Z_{2}+Z_{1}\right)+\left\langle Y_{k}, \nabla_{J Y} \mathbf{J X}_{i}\right\rangle\left(Z_{2}-J Z_{1}\right) \\
& -\left\langle\mathbf{X}_{\mathbf{k}}, \nabla \mathbf{J} \mathbf{Y}_{\mathbf{j}} \mathbf{J X}_{\mathbf{i}}\right\rangle \mathbf{Z}_{\mathbf{s}}-\left\langle\mathbf{X}_{\mathbf{k}}, \nabla \mathbf{J} \mathbf{Y}_{\mathbf{j}} \mathbf{X}_{\mathbf{i}}\right\rangle \mathbf{J Z} \mathbf{Z}_{\mathbf{s}} \\
& -\sum_{\alpha>4}\left(\left\langle A_{\alpha} \mathbf{Y}_{\mathbf{k}}, \nabla J Y_{j} J_{X_{i}}\right\rangle Z_{\alpha}+\left\langle\Lambda_{\alpha \star} \mathbf{Y}_{\mathbf{k}}, \nabla J Y_{j} \mathbf{J X}_{\mathbf{i}}\right\rangle \mathbf{J Z}_{\alpha}\right) \\
& -\left\langle\mathbf{X}_{\mathbf{i}}, \boldsymbol{\nabla}_{\mathbf{J Y}} \mathbf{Y}_{\mathbf{j}}\right\rangle\left(\mathrm{JZ}_{\mathbf{2}}-\mathbf{Z}_{\mathbf{l}}\right)-\left\langle\mathbf{X}_{\mathbf{i}}, \boldsymbol{\nabla J}_{\mathbf{J Y}} \mathbf{J Y}_{\mathbf{k}}\right\rangle\left(\mathrm{Z}_{\mathbf{2}}+\mathrm{JZ}_{\mathbf{l}}\right) \\
& -\left\langle\mathbf{Y}_{\mathbf{i}}, \boldsymbol{\nabla}_{\mathbf{J Y}} \mathbf{J Y}_{\mathbf{j}}\right\rangle \mathbf{Z}_{\mathbf{3}}-\left\langle\mathbf{Y}_{\mathbf{i}}, \boldsymbol{\nabla}_{\mathbf{J Y}} \mathbf{Y}_{\mathbf{j}} \mathbf{Y}_{\mathbf{k}}\right\rangle \mathbf{J Z}_{\mathbf{3}} \\
& -\sum_{\alpha \geq 4}\left(\left\langle A_{\alpha} X_{i}, \nabla J Y_{j} J_{\mathbf{k}_{k}}\right\rangle Z_{\alpha}+\left\langle\Lambda_{\alpha_{\star}} X_{i}, \nabla J Y_{j} J_{\mathbf{k}_{k}}\right\rangle J Z_{\alpha}\right) .
\end{aligned}
$$

Since the equation of Codazzi gives

$$
\left(\bar{\nabla}_{\mathbf{X}_{\mathbf{i}} \boldsymbol{\sigma}}\right)\left(\mathbf{J} \mathbf{Y}_{\mathbf{j}}, \mathbf{J} \mathbf{Y}_{\mathbf{k}}\right)=\left(\bar{\nabla}_{\mathbf{J Y}} \mathbf{j}_{\mathbf{j}} \boldsymbol{\sigma}\right)\left(\mathbf{X}_{\mathbf{i}}, \mathbf{J} \mathbf{Y}_{\mathbf{k}}\right) \text {, }
$$


the $Z_{1}$-components of both sides of (3.50) yield

$$
2\left\langle J Y_{k}, \nabla X_{i} Y_{j}\right\rangle=\delta_{j k} \theta_{22}\left(X_{i}\right),
$$

where we used (3.39), (3.40) and the fact that $X_{i}$ and $Y_{k}$ are orthogonal. Similarly, by comparing the $\mathrm{JZ}_{1}-, \mathrm{JZ}_{2}-$, and $\mathrm{JZ}_{3}$-components of (3.50), we may also obtain

$$
\begin{aligned}
& 2\left\langle J Y_{k}, \nabla J Y_{j} X_{i}\right\rangle=\delta_{i k} \theta_{1}\left(J Y_{j}\right)+\underset{\alpha \geqslant 4}{\sum}\left\langle A_{\alpha} X_{i}, Y_{k}\right\rangle \theta_{1 \alpha}\left(J Y_{j}\right), \\
& 2\left\langle Y_{k}, \nabla J Y_{j} X_{i}\right\rangle=\delta_{i k \theta_{23}}\left(J Y_{j}\right)+\underset{\alpha i 4}{\sum}\left\langle A_{\alpha} X_{i}, Y_{k}\right\rangle \theta_{2 \alpha}\left(J Y_{j}\right), \\
& -\delta_{j k_{13} \boldsymbol{\theta}_{\mathbf{3}}}\left(\mathbf{X}_{\mathbf{i}}\right)+\left\langle J \mathbf{X}_{\mathbf{k}}, \boldsymbol{\nabla}_{\mathbf{X}_{\mathbf{i}}} \mathbf{Y}_{\mathbf{j}}\right\rangle+\left\langle J \mathbf{X}_{\mathbf{j}}, \boldsymbol{\nabla}_{\mathbf{X}_{\mathbf{i}}} \mathbf{Y}_{\mathbf{k}}\right\rangle \\
& =\sum_{\alpha \geq 4}\left\langle A_{\alpha} X_{i}, Y_{k}\right\rangle \theta_{\alpha_{3}}\left(J Y_{j}\right)-\left\langle X_{k}, \nabla_{J Y} \mathbf{X}_{j} X_{i}\right\rangle-\left\langle Y_{i}, \nabla_{J Y} Y_{j} Y_{k}\right\rangle,
\end{aligned}
$$

where we used (3.51) to derive (3.53).

Since $A_{\alpha} A_{3}+A_{3} A_{\alpha}=0$ for $\alpha \gg 4$, Lemma 5 implies

$$
\left\langle\boldsymbol{\Lambda}_{\alpha} \mathbf{X}_{\mathbf{i}}, \mathbf{Y}_{\mathbf{k}}\right\rangle=-\left\langle\boldsymbol{\Lambda}_{\boldsymbol{\alpha}} \mathbf{X}_{\mathbf{k}}, \mathbf{Y}_{\mathbf{i}}\right\rangle \text {. }
$$

Therefore, (3.52) and (3.53) yield

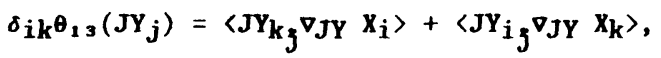

$$
\begin{aligned}
& \delta_{i k} \boldsymbol{\theta}_{23}\left(J Y_{j}\right)=\left\langle Y_{k}, \nabla J Y_{j} X_{i}\right\rangle+\left\langle Y_{i}, \nabla_{J Y} \mathbf{X}_{\mathbf{k}}\right\rangle \text {. }
\end{aligned}
$$

Furthermore, from (3.55), we see that the left-hand side of (3.54) is symmetric with respect to the indices $j$ and $k$ and the right-hand side is skew-symmetric with respect to $j$ and $k$, thus we obtain

$$
\begin{aligned}
& \boldsymbol{\delta}_{\mathbf{j k} \boldsymbol{\theta}_{\mathbf{3}}}\left(\mathbf{X}_{\mathbf{i}}\right)=\left\langle\mathbf{J} \mathbf{Y}_{\mathbf{j}}, \boldsymbol{\nabla}_{\mathbf{X}_{\mathbf{i}}} \mathbf{X}_{\mathbf{k}}\right\rangle+\left\langle\mathbf{J Y}_{\mathbf{k}}, \boldsymbol{\nabla}_{\mathbf{X}_{\mathbf{i}}} \mathbf{X}_{\mathbf{j}}\right\rangle, \\
& \left\langle\nabla_{J} Y_{j} Y_{i}, Y_{k}\right\rangle-\left\langle\nabla J Y_{j} X_{i}, X_{k}\right\rangle=\sum_{\alpha=4}\left\langle A_{\alpha} X_{i}, Y_{k}\right\rangle \theta_{3 \alpha}\left(J Y_{j}\right) .
\end{aligned}
$$

From (3.51) (respectively, (3.52), (3.53) and (3.59)), we obtain (3.42) for $T$ in $V^{\prime}$ (respectively, (3.43), (3.44), and (3.45) for $T$ in JV*). By using the same method, we may obtain (3.41) - (3.45) for all $T$ in $T N$. (The computation is long, but straight-forward).

In the following, we denote by $R$ and $R^{\perp}$ the Riemann curvature tensor and the normal curvature tensor of the leaf $N$.

LEMMA 7. Under the hypothesis and the notations of Lemma 5, we have

$$
\begin{aligned}
& 2 R\left(X_{2}, Y_{2} ; Y_{2}, X_{2}\right)+2\left\langle\nabla_{1} Y_{1}, \nabla X_{2} X_{1}\right\rangle-2\left\langle\nabla X_{1} Y_{1}, \nabla_{Y_{1}} X_{1}\right\rangle \\
& =R^{+}\left(X_{1}, Y_{1} ; Z_{3}, Z_{2}\right)+\left\langle D_{Y_{2}} Z_{3}, D_{X_{2}} Z_{2}\right\rangle-\left\langle D_{X_{1}} Z_{3}, D_{Y_{1}} Z_{2}\right\rangle
\end{aligned}
$$

PROOF. From Lemma 5, we have $\left\langle A_{\alpha} X_{1}, Y_{1}\right\rangle=\left\langle A_{\alpha} X_{1}, A_{3} X_{1}\right\rangle=\left\langle X_{1}, A_{\alpha} A_{3} A_{1}\right\rangle=0$ for $\alpha$ 4. Thus Lemma 6 implies $2\left\langle\nabla_{\mathrm{T}} \mathrm{Y}_{1}, \mathrm{X}_{2}\right\rangle=\theta_{32}(\mathrm{~T})=\left\langle\mathrm{D}_{\mathrm{T}} \mathrm{Z}_{3}, \mathrm{Z}_{2}\right\rangle$, from which we obtain (3.60).

(Q.E.D.)

\section{PROOF OF THEOREM 1.}

Under the hypothesis of Theorem 1 , if $M$ is non-proper, Lemma 3 implies $p=$ $\operatorname{dim}_{R} \sigma^{+} \rightarrow 3$.

If $p \geq 4$, then Lemmas 5 and 6 imply that, for $i \geq 2$, we have 


$$
\begin{aligned}
2\left\langle\nabla_{\mathrm{T}} \mathrm{Y}_{1}, \mathrm{X}_{\mathrm{i}}\right\rangle & =\sum_{\alpha \geqslant 4}\left\langle A_{\alpha} \mathrm{X}_{\mathrm{i}}, \mathrm{Y}_{1}\right\rangle \boldsymbol{\theta}_{\alpha 2}(\mathrm{~T}) \\
= & \sum_{\alpha \geqslant 4}\left\langle\mathrm{~A}_{\alpha A_{3}} \mathrm{X}_{1}, \mathrm{X}_{\mathrm{i}}\right\rangle \boldsymbol{\theta}_{\alpha 2}(\mathrm{~T})=\sum_{\alpha \geqslant 4}\left\langle\mathrm{X}_{\alpha-2}, \mathrm{X}_{\mathrm{i}}\right\rangle \boldsymbol{\theta}_{\alpha 2}(\mathrm{~T}) .
\end{aligned}
$$

Thus, we have $2\left\langle\nabla_{\mathrm{T}} \mathrm{Y}_{1}, \mathrm{X}_{\mathrm{i}}\right\rangle=\theta_{\mathrm{i}+22}(\mathrm{~T}), \quad \mathrm{i}=2, \ldots, \mathrm{r} . \quad$ Similarly, we have $2\left\langle\nabla_{\mathrm{T}} \mathrm{X}_{1}, \mathrm{Y}_{\mathrm{i}}\right\rangle=\theta_{\mathrm{i}+22}(\mathrm{~T}), \quad \mathrm{i}=2, \ldots, \mathrm{r}$. Thus, by applying Lemma 6 , we may obtain

$$
\begin{aligned}
& 2\left\langle\nabla_{Y_{1}} Y_{1}, \nabla_{X_{1}} X_{1}\right\rangle-2\left\langle\nabla_{X_{1}} Y_{1}, \nabla_{Y_{1}} X_{1}\right\rangle \\
& =\sum_{i=2}^{P-2} \theta_{i+22}\left(Y_{1}\right)\left[\left\langle\nabla_{X_{1}} X_{1}, X_{i}\right\rangle-\left\langle\nabla_{X_{1}} Y_{1}, Y_{i}\right\rangle\right] \\
& \quad+\sum_{i=2}^{P-2} \theta_{i+22}\left(X_{1}\right)\left[\left\langle\nabla_{Y_{1}} Y_{1}, Y_{i}\right\rangle-\left\langle\nabla_{Y_{1}} X_{1}, X_{i}\right\rangle\right] \\
& \quad+2 \theta_{12}\left(X_{1}\right)\left\langle\nabla_{Y_{1}} X_{1}, J Y_{1}\right\rangle+2 \theta_{21}\left(Y_{1}\right)\left\langle\nabla_{X_{1}} X_{1}, J Y_{1}\right\rangle .
\end{aligned}
$$

Therefore, by applying Lemma 6 again, we may find

$$
2\left\langle\nabla_{Y_{1}} Y_{1}, \nabla_{X_{2}} X_{2}\right\rangle-2\left\langle\nabla_{X_{1}} Y_{1}, \nabla_{Y_{2}} X_{1}\right\rangle=\left\langle D_{X_{1}} Z_{2}, D_{Y_{2}} Z_{3}\right\rangle-\left\langle D_{Y_{1}} Z_{2}, D_{X_{1}} Z_{3}\right\rangle
$$

Combining (4.1) with (3.60) of Lemena 7 , we get

$$
2 R\left(X_{1}, Y_{1} ; Y_{1}, X_{1}\right)=R^{\perp}\left(X_{1}, Y_{1} ; Z_{3}, Z_{2}\right) \text {. }
$$

From (2.7), (3.46), (3.47), Lemma 5 and the equation of Gauss, we may find

$$
R\left(X_{1}, Y_{1} ; Y_{1}, X_{1}\right)=-2 \text {. }
$$

On the other hand, (2.7), the equation of Ricci, Lemma 1 and Lemma 5 give

$$
R^{\perp}\left(X_{1}, Y_{1} ; Z_{3}, Z_{2}\right)=2\left\langle A_{2} X_{1}, X_{2}\right\rangle=2 \text {. }
$$

Equations (4.2), (4.3) and (4.4) give a contradiction. If $p=3$, then, by (3.27) and the equation of Codazzi, we may obtain (3.41) - (3.45) in such forms that the summation terms in (3.43) - (3.45) were disappeared. By applying these equations, we may obtain a contradiction in a similar way.

(Q.E.D.)

REMARK. For a CR-submanifold $M$ of a Kaehler manifold, the condition that $M$ is mixed-foliate is equivalent to $\mathrm{AP}=-\mathrm{PA}$.

\section{REFBRENCES}

1. CHEN, B.Y.: CR-8ubmanifolds of a Kaehler manifold, I, J. Differential Goometry, 16 (1981), 305-322; - II, ibid, 16 (1981), 493-509.

2. BEJANCU, A.: Goometry of CR-submanifolds, Reidel Publishing Co., Dordrecht, 1986.

3. BLAIR, D.E. and CHEN, B.Y.: On CR-submanifolds of Hermitian manifolds, Israel J. Math., 34 (1979), 353-363.

4. BEJANCU, A., KON, M. and YANO, K.: CR-submanifolds of a complex space form, J. Differential Goometry, 16 (1981), 137-145. 


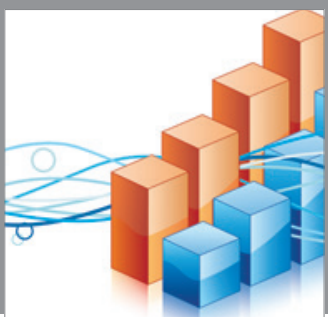

Advances in

Operations Research

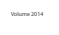

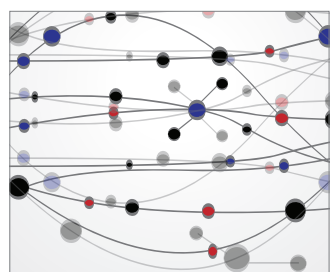

\section{The Scientific} World Journal
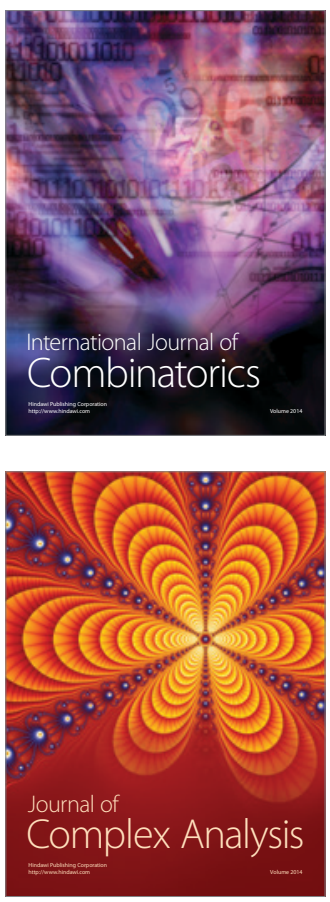

International Journal of

Mathematics and

Mathematical

Sciences
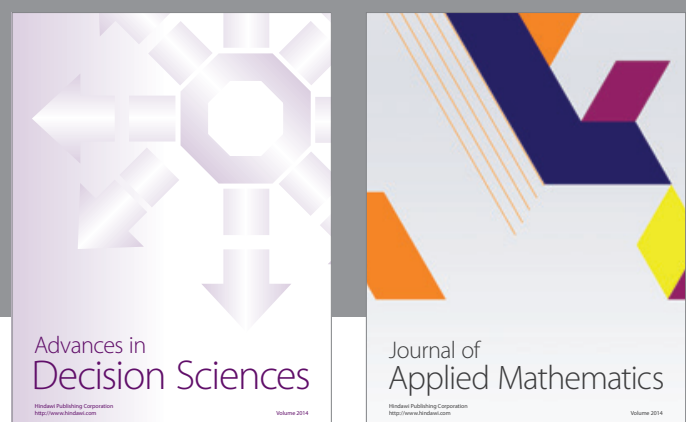

Journal of

Applied Mathematics
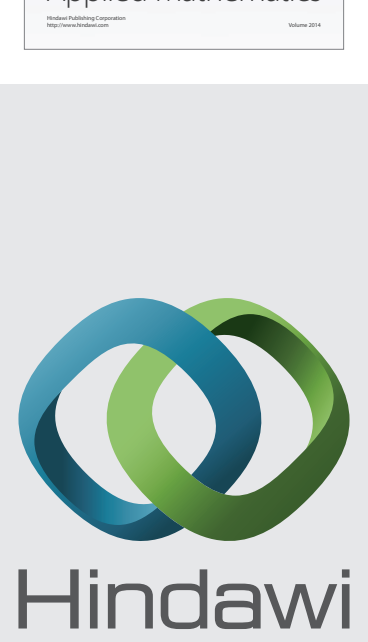

Submit your manuscripts at http://www.hindawi.com
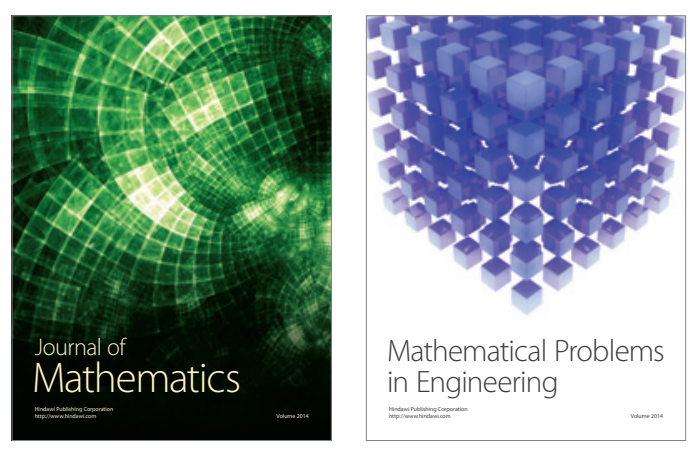

Mathematical Problems in Engineering
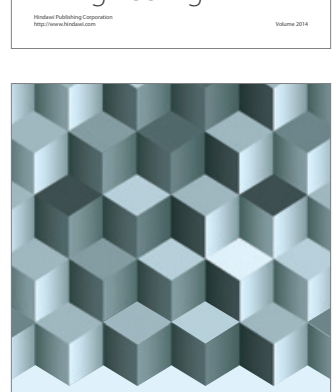

Journal of

Function Spaces
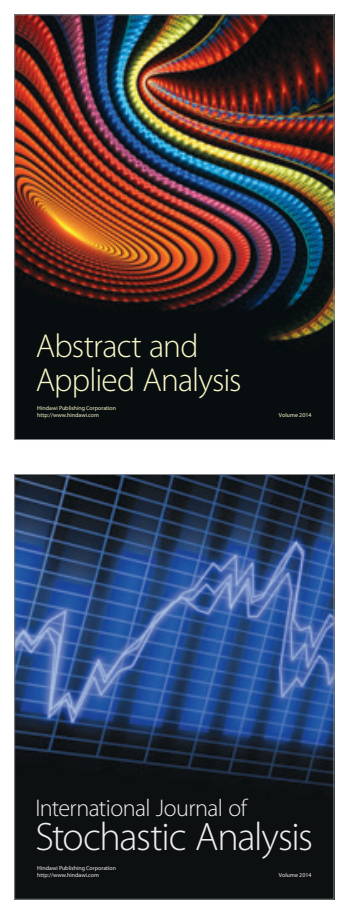

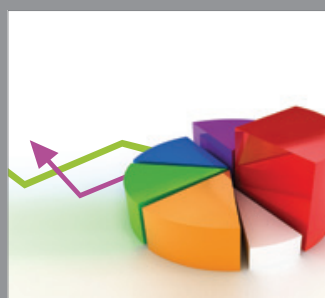

ournal of

Probability and Statistics

Promensencen
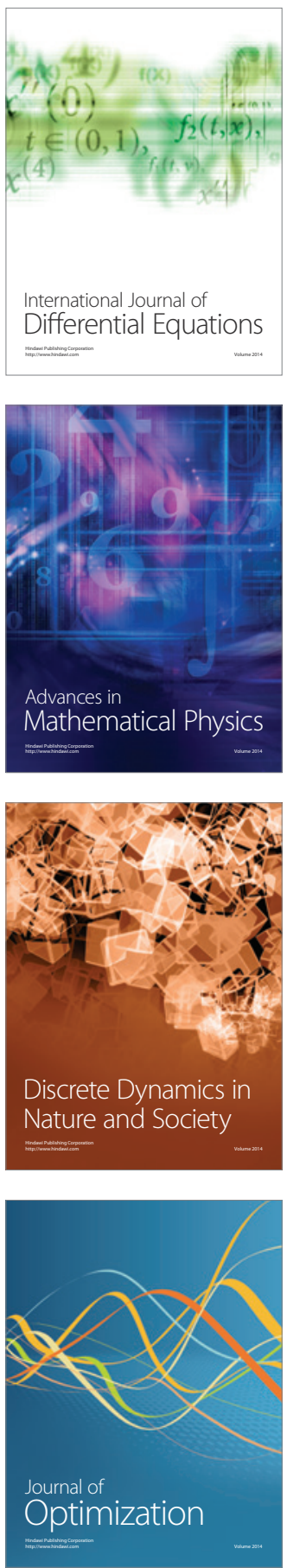